Special Issue: 25 years of the Mizar Mathematical Library

FORMALIZED MATHEMATICS

Vol. 22, No. 2, Pages $105-110,2014$

DE GRUYTER

DOI: 10.2478 /forma-2014-0012

degruyter.com/view/j/forma

\title{
Lagrange's Four-Square Theorem
}

\author{
Yasushige Watase \\ Suginami-ku Matsunoki 6 \\ 3-21 Tokyo, Japan
}

Summary. This article provides a formalized proof of the so-called "the four-square theorem", namely any natural number can be expressed by a sum of four squares, which was proved by Lagrange in 1770. An informal proof of the theorem can be found in the number theory literature, e.g. in [14], [1] or [23].

This theorem is item \#19 from the "Formalizing 100 Theorems" list maintained by Freek Wiedijk at http://www.cs.ru.nl/F.Wiedijk/100/.

MSC: 11P99 03B35

Keywords: Lagrange's four-square theorem

MML identifier: LAGRA4SQ, version: 8.1.03 5.23.1207

The notation and terminology used in this paper have been introduced in the following articles: [19], [2], [7, [6], [12], 8], [9], 21], [17, [4], [15], [16], [5], [10], [13], 24], 25], 22], and [11].

\section{Preliminaries}

Let $n$ be a natural number. We say that $n$ is a sum of four squares if and only if

(Def. 1) There exist natural numbers $n_{1}, n_{2}, n_{3}, n_{4}$ such that $n=n_{1}^{2}+n_{2}^{2}+$ $n_{3}^{2}+n_{4}^{2}$.

Note that there exists a natural number which is a sum of four squares.

Let $y$ be an integer object. Let us note that $|y|$ is natural.

Now we state the proposition:

(1) Let us consider natural numbers $n_{1}, n_{2}, n_{3}, n_{4}, m_{1}, m_{2}, m_{3}, m_{4}$. Then $\left(n_{1}^{2}+n_{2}^{2}+n_{3}^{2}+n_{4}^{2}\right) \cdot\left(m_{1}^{2}+m_{2}^{2}+m_{3}^{2}+m_{4}^{2}\right)=\left(n_{1} \cdot m_{1}-n_{2} \cdot m_{2}-\right.$ $\left.n_{3} \cdot m_{3}-n_{4} \cdot m_{4}\right)^{2}+\left(n_{1} \cdot m_{2}+n_{2} \cdot m_{1}+n_{3} \cdot m_{4}-n_{4} \cdot m_{3}\right)^{2}+\left(n_{1} \cdot m_{3}-\right.$ $\left.n_{2} \cdot m_{4}+n_{3} \cdot m_{1}+n_{4} \cdot m_{2}\right)^{2}+\left(n_{1} \cdot m_{4}+n_{2} \cdot m_{3}-n_{3} \cdot m_{2}+n_{4} \cdot m_{1}\right)^{2}$. 
Let $m, n$ be natural numbers. Let us note that $m \cdot n$ is a sum of four squares and there exists a prime natural number which is odd.

From now on $i, j, k, v, w$ denote natural numbers, $j_{1}, j_{2}, m, n, s, t, x, y$ denote integers, and $p$ denotes an odd prime natural number.

Let us consider $p$. The functor $\operatorname{ModMap}(p)$ yielding a function from $\mathbb{Z}$ into $\mathbb{Z}_{p}$ is defined by

(Def. 2) Let us consider an element $x$ of $\mathbb{Z}$. Then it $(x)=x \bmod p$.

Let us consider $v$. The functor $\operatorname{Lag} 4 \mathrm{SqF}(v)$ yielding a finite sequence of elements of $\mathbb{Z}$ is defined by

(Def. 3) (i) len $i t=v$, and

(ii) for every natural number $i$ such that $i \in \operatorname{dom} i t$ holds $i t(i)=(i-1)^{\mathbf{2}}$.

The functor $\operatorname{Lag} 4 \operatorname{SqG}(v)$ yielding a finite sequence of elements of $\mathbb{Z}$ is defined by

(Def. 4) (i) len $i t=v$, and

(ii) for every natural number $i$ such that $i \in \operatorname{dom} i t$ holds $i t(i)=-1-$ $(i-1)^{2}$.

Now we state the propositions:

(2) $\operatorname{Lag} 4 \mathrm{SqF}(v)$ is one-to-one.

(3) $\operatorname{Lag} 4 \mathrm{SqG}(v)$ is one-to-one.

In the sequel $a$ denotes a real number and $b$ denotes an integer.

Let us consider an odd prime natural number $p$, a natural number $s, j_{1}$, and $j_{2}$. Now we state the propositions:

(4) If $2 \cdot s=p+1$ and $j_{1}, j_{2} \in \operatorname{rng} \operatorname{Lag} 4 \operatorname{SqF}(s)$, then $j_{1}=j_{2}$ or $j_{1} \bmod p \neq$ $j_{2} \bmod p$. Proof: Consider $s$ such that $p+1=2 \cdot s$. For every integers $j_{1}, j_{2}$ such that $j_{1}, j_{2} \in \operatorname{rng} \operatorname{Lag} 4 \operatorname{SqF}(s)$ and $j_{1} \neq j_{2}$ holds $j_{1} \bmod p \neq j_{2} \bmod p$ by [21, (3), (55)], [16, (80)], [18, (22)].

(5) If $2 \cdot s=p+1$ and $j_{1}, j_{2} \in \operatorname{rng} \operatorname{Lag} 4 \operatorname{SqG}(s)$, then $j_{1}=j_{2}$ or $j_{1} \bmod p \neq$ $j_{2} \bmod p$. Proof: Consider $s$ such that $p+1=2 \cdot s$. For every $j_{1}$ and $j_{2}$ such that $j_{1}, j_{2} \in \operatorname{rng} \operatorname{Lag} 4 \operatorname{SqG}(s)$ and $j_{1} \neq j_{2}$ holds $j_{1} \bmod p \neq j_{2} \bmod p$ by [21, (3), (55)], [16, (80)], [20, (7)].

\section{Any Prime Number can be Expressed as a Sum of Four Squares}

Now we state the propositions:

(6) There exist natural numbers $x_{1}, x_{2}, x_{3}, x_{4}, h$ such that

(i) $0<h<p$, and

(ii) $h \cdot p=x_{1}^{2}+x_{2}^{2}+x_{3}^{2}+x_{4}^{2}$. 
Proof: Consider $s$ such that $2 \cdot s=p+1$. Set $f=\operatorname{Lag} 4 \operatorname{SqF}(s)$. Set $g=\operatorname{Lag} 4 \operatorname{SqG}(s) . f$ is one-to-one. $g$ is one-to-one. $\operatorname{rng} f$ misses $\operatorname{rng} g$. $\overline{\overline{\operatorname{rng}\left(g^{\frown f)}\right.}}=p+1$ by [2, (70)], [6, (57), (31)], [3, (35), (36)]. Set $A=$ $\operatorname{dom}\left(\operatorname{ModMap}(p)\left\lceil\operatorname{rng}\left(g^{\frown} f\right)\right)\right.$. Set $B=\operatorname{rng}\left(\operatorname{ModMap}(p)\left\lceil\operatorname{rng}\left(g^{\frown} f\right)\right)\right.$. Define $\mathcal{P}$ [object, object] $\equiv$ there exists an element $m_{1}$ of $\mathbb{Z}$ such that $\$_{1} \in A$ and $\$_{2}=m_{1}$ and $\left(\operatorname{ModMap}(p)\left\lceil\operatorname{rng}\left(g^{\frown} f\right)\right)\left(\$_{1}\right)=m_{1}\right.$. For every object $x$ such that $x \in A$ there exists an object $y$ such that $y \in B$ and $\mathcal{P}[x, y]$ by $[\underline{8}$, (3)]. Consider $h$ being a function from $A$ into $B$ such that for every object $x$ such that $x \in A$ holds $\mathcal{P}[x, h(x)]$ from [9, Sch. 1]. Consider $m_{1}, m_{2}$ being objects such that $m_{1} \in A$ and $m_{2} \in A$ and $m_{1} \neq m_{2}$ and $h\left(m_{1}\right)=h\left(m_{2}\right)$. If $m_{1} \in \operatorname{rng} f$, then $m_{2} \in \operatorname{rng} g$. If $m_{1} \in \operatorname{rng} g$, then $m_{2} \in \operatorname{rng} f$. There exist natural numbers $x_{1}, x_{2}, x_{3}, x_{4}, h$ such that $h>0$ and $h<p$ and $h \cdot p=x_{1}^{2}+x_{2}^{2}+x_{3}^{2}+x_{4}^{2}$ by [20, (7)], [21, (3)].

(7) Let us consider natural numbers $x_{1}, h$. Suppose $1<h$. Then there exists an integer $y_{1}$ such that

(i) $x_{1} \bmod h=y_{1} \bmod h$, and

(ii) $-h<2 \cdot y_{1} \leqslant h$, and

(iii) $x_{1}^{2} \bmod h=y_{1}^{2} \bmod h$.

Proof: Consider $q_{1}, r_{1}$ being integers such that $x_{1}=h \cdot q_{1}+r_{1}$ and $0 \leqslant r_{1}$ and $r_{1}<h$. There exists an integer $y_{1}$ such that $x_{1} \bmod h=y_{1} \bmod h$ and $-h<2 \cdot y_{1} \leqslant h$ and $x_{1}^{2} \bmod h=y_{1}^{2} \bmod h$ by [21, (3)], [18, (23)].

(8) Let us consider natural numbers $i_{1}, i_{2}, c$. If $i_{1} \leqslant c$ and $i_{2} \leqslant c$, then $i_{1}+i_{2}<2 \cdot c$ or $i_{1}=c$ and $i_{2}=c$.

(9) Let us consider natural numbers $i_{1}, i_{2}, i_{3}, i_{4}, c$. Suppose

(i) $i_{1} \leqslant c$, and

(ii) $i_{2} \leqslant c$, and

(iii) $i_{3} \leqslant c$, and

(iv) $i_{4} \leqslant c$.

Then

(v) $i_{1}+i_{2}+i_{3}+i_{4}<4 \cdot c$, or

(vi) $i_{1}=c$ and $i_{2}=c$ and $i_{3}=c$ and $i_{4}=c$.

The theorem is a consequence of (8).

Let us consider natural numbers $x_{1}, h$ and an integer $y_{1}$. Now we state the propositions:

(10) Suppose $1<h$ and $x_{1} \bmod h=y_{1} \bmod h$ and $-h<2 \cdot y_{1}$ and $\left(2 \cdot y_{1}\right)^{2}=$ $h^{2}$. Then

(i) $2 \cdot y_{1}=h$, and 
(ii) there exists a natural number $m_{1}$ such that $2 \cdot x_{1}=\left(2 \cdot m_{1}+1\right) \cdot h$.

(11) If $1<h$ and $x_{1} \bmod h=y_{1} \bmod h$ and $y_{1}=0$, then there exists an integer $m_{1}$ such that $x_{1}=h \cdot m_{1}$.

Now we state the proposition:

(12) Let us consider an odd prime number $p$ and natural numbers $x_{1}, x_{2}, x_{3}$, $x_{4}, h$. Suppose

(i) $1<h<p$, and

(ii) $h \cdot p=x_{1}^{2}+x_{2}^{2}+x_{3}^{2}+x_{4}^{2}$.

Then there exist integers $y_{1}, y_{2}, y_{3}, y_{4}$ and there exists a natural number $r$ such that $0<r<h$ and $r \cdot p=y_{1}{ }^{2}+y_{2}{ }^{2}+y_{3}{ }^{2}+y_{4}{ }^{2}$. The theorem is a consequence of (7), (9), (10), and (11).

Let us consider a prime number $p$. Now we state the propositions:

(13) If $p$ is even, then $p=2$.

(14) There exist natural numbers $x_{1}, x_{2}, x_{3}, x_{4}$ such that $p=x_{1}{ }^{2}+x_{2}{ }^{2}+$ $x_{3}^{2}+x_{4}^{2}$.

Now we state the proposition:

(15) Let us consider prime numbers $p_{1}, p_{2}$. Then there exist natural numbers $x_{1}, x_{2}, x_{3}, x_{4}$ such that $p_{1} \cdot p_{2}=x_{1}{ }^{2}+x_{2}{ }^{2}+x_{3}{ }^{2}+x_{4}{ }^{2}$. The theorem is a consequence of (14).

Let $p_{1}, p_{2}$ be prime numbers. Let us observe that $p_{1} \cdot p_{2}$ is a sum of four squares.

Now we state the proposition:

(16) Let us consider a prime number $p$ and a natural number $n$. Then there exist natural numbers $x_{1}, x_{2}, x_{3}, x_{4}$ such that $p^{n}=x_{1}{ }^{2}+x_{2}{ }^{2}+x_{3}{ }^{2}+x_{4}{ }^{2}$. Proof: Define $\mathcal{P}$ [natural number] $\equiv$ there exist natural numbers $x_{1}, x_{2}$, $x_{3}, x_{4}$ such that $p^{\$_{1}}=x_{1}{ }^{2}+x_{2}{ }^{2}+x_{3}{ }^{2}+x_{4}{ }^{2}$. For every natural number $n$ such that $\mathcal{P}[n]$ holds $\mathcal{P}[n+1]$ by (14), [7, (75)], [16, (6)]. $\mathcal{P}[0]$ by [16, (4)]. For every natural number $n, \mathcal{P}[n]$ from [4, Sch. 2].

Let $p$ be a prime number and $n$ be a natural number. Observe that $p^{n}$ is a sum of four squares.

\section{Proof of LAGRANGe's theorem}

Now we state the proposition:

(17) Let us consider a non zero natural number $n$. Then there exist natural numbers $x_{1}, x_{2}, x_{3}, x_{4}$ such that $\prod \operatorname{PPF}(n)=x_{1}{ }^{2}+x_{2}{ }^{2}+x_{3}{ }^{2}+x_{4}{ }^{2}$. Proof: Define $\mathcal{P}$ [natural number] $\equiv$ for every non zero natural number $n$ such that $\overline{\overline{\text { support } \operatorname{PPF}(n)}}=\$_{1}$ there exist natural numbers $x_{1}, x_{2}, x_{3}$, $x_{4}$ such that $\prod \operatorname{PPF}(n)=x_{1}{ }^{2}+x_{2}{ }^{2}+x_{3}{ }^{2}+x_{4}{ }^{2}$. $\mathcal{P}[0]$ by [15, (20)]. For 
every natural number $k$ such that $\mathcal{P}[k]$ holds $\mathcal{P}[k+1]$ by [15, (34), (28), (25)]. For every natural number $k, \mathcal{P}[k]$ from [4, Sch. 2].

Now we state the proposition:

\section{(18) LAGRANGE'S FOUR-SQUARE THEOREM:}

Let us consider a natural number $n$. Then there exist natural numbers $x_{1}, x_{2}, x_{3}, x_{4}$ such that $n=x_{1}^{2}+x_{2}^{2}+x_{3}^{2}+x_{4}^{2}$. The theorem is a consequence of (17).

One can verify that every natural number is a sum of four squares.

\section{REFERENCES}

[1] Alan Baker. A Concise Introduction to the Theory of Numbers. Cambridge University Press, 1984.

[2] Grzegorz Bancerek. Cardinal numbers. Formalized Mathematics, 1(2):377-382, 1990.

[3] Grzegorz Bancerek. Cardinal arithmetics. Formalized Mathematics, 1(3):543-547, 1990.

[4] Grzegorz Bancerek. The fundamental properties of natural numbers. Formalized Mathematics, 1(1):41-46, 1990.

[5] Grzegorz Bancerek. The ordinal numbers Formalized Mathematics, 1(1):91-96, 1990.

[6] Grzegorz Bancerek and Krzysztof Hryniewiecki. Segments of natural numbers and finite sequences Formalized Mathematics, 1(1):107-114, 1990.

[7] Czesław Byliński. The complex numbers Formalized Mathematics, 1(3):507-513, 1990.

[8] Czesław Byliński. Functions and their basic properties Formalized Mathematics, 1(1): 55-65, 1990.

[9] Czesław Byliński. Functions from a set to a set. Formalized Mathematics, 1(1):153-164, 1990.

[10] Czesław Byliński. Partial functions. Formalized Mathematics, 1(2):357-367, 1990.

[11] Czesław Byliński. Some basic properties of sets. Formalized Mathematics, 1(1):47-53, 1990.

[12] Agata Darmochwał. Finite sets Formalized Mathematics, 1(1):165-167, 1990.

[13] Yoshinori Fujisawa, Yasushi Fuwa, and Hidetaka Shimizu. Public-key cryptography and Pepin's test for the primality of Fermat numbers Formalized Mathematıcs, $7(\mathbf{2}): 317-321$, 1998.

[14] G.H. Hardy and E.M. Wright. An Introduction to the Theory of Numbers. Oxford University Press, 1980.

[15] Artur Korniłowicz and Piotr Rudnicki. Fundamental Theorem of Arithmetic Formalized Mathematics, 12(2):179-186, 2004.

[16] Rafał Kwiatek. Factorial and Newton coefficients Formalized Mathematics, 1(5):887-890 1990.

[17] Rafał Kwiatek and Grzegorz Zwara. The divisibility of integers and integer relatively primes. Formalized Mathematics, 1(5):829-832, 1990.

[18] Xiquan Liang, Li Yan, and Junjie Zhao. Linear congruence relation and complete residue systems. Formalized Mathematics, 15(4):181-187, 2007. doi:10.2478/v10037-007-0022-7.

[19] Piotr Rudnicki and Andrzej Trybulec. Abian's fixed point theorem. Formalized Mathematics, 6(3):335-338, 1997.

[20] Christoph Schwarzweller. Modular integer arithmetic. Formalized Mathematics, 16(3): 247-252, 2008. doi $10.2478 /$ v10037-008-0029-8

[21] Michał J. Trybulec. Integers. Formalized Mathematics, 1(3):501-505, 1990.

[22] Zinaida Trybulec. Properties of subsets. Formalized Mathematics, 1(1):67-71, 1990.

[23] Hideo Wada. The World of Numbers (in Japanese). Iwanami Shoten, 1984. 
[24] Edmund Woronowicz. Relations and their basic properties. Formalized Mathematics, 1 (1):73-83, 1990.

[25] Edmund Woronowicz. Relations defined on sets Formalized Mathematics, 1(1):181-186, 1990.

Received June 4, 2014 